\title{
Pared Membranosa de los Bronquios: Propuesta de Inclusión en la Terminología Anatómica
}

\author{
Membranous Wall Bronchus: Proposal for Inclusion in the Terminologia Anatomica
}

\author{
Jorge Eduardo Duque Parra*,**; John Barco Ríos* \& Juan Fernando Vélez García***
}

\begin{abstract}
DUQUE, P. J. E.; BARCO, R. J. \& VÉLEZ, G. J. F. Pared membranosa de los bronquios: propuesta de inclusión en la Terminología Anatómica. Int. J. Morphol., 32(4):1388-1390, 2014.

RESUMEN: Los bronquios principales son dos tubos respiratorios que se originan de la parte inferior de la tráquea y se proyectan a cada pulmón, permitiendo el paso de aire hacia y desde los bronquíolos. El objetivo de este trabajo fue verificar la inclusión como término morfológico de la pared posterior de los bronquios principales en la Terminologia Anatomica y en los textos de enseñanza de la anatomía macroscópica. Se revisó la Terminologia Anatomica y se comparó con diversos textos y artículos de enseñanza de la anatomía macroscópica, con el fin de verificar si hay descripciones de la pared posterior de los bronquios principales y si ésta se encuentra nominada. No está incluido en la Terminologia Anatomica ningún término para nominar la pared posterior de los bronquios principales, por lo que se propone incluir el término pared membranosa para tales bronquios en la Terminologia Anatomica.
\end{abstract}

PALABRAS CLAVE: Bronquios principales; Pared membranosa; Terminologia Anatómica.

\section{INTRODUCCIÓN}

Aunque la terminología per se es incapaz de expresar la verdad objetiva sobre la realidad, nos permite, al menos en parte, aproximarnos a dicha verdad, para lo cual los seres humanos hemos desarrollado convenciones lingüísticas (Duque et al., 2010) que nos aproximan más a dicha verdad. En nuestro caso, una terminología para la descripción anatómica con el fin de poder solucionar cuestiones terminológicas propias de una segmentación y fragmentación de las ciencias médicas y biológicas (Losardo et al., 2010). Los lenguajes siempre cambian y dichos cambios tienen gran visibilidad cuando la emergencia de una nueva unidad describe un concepto o una nueva realidad llega a ser aparente (Cabré Castellví et al., 2012), como en el campo morfológico, y la más importante razón detrás de la renovación del léxico es la necesidad de cambiar el lenguaje para adaptarlo al medioambiente cambiante (Cabré Castellví et al.). Esto facilita el diálogo y la comprensión de aquella realidad que deseamos expresar, a pesar de cierta carga de subjetividad intrínseca. De igual manera, en el campo de la morfología fue necesario implementar una terminología apropiada para la descripción anatómica que permitiera una correcta comunicación entre la comunidad científica (Du- que et al., 2002), concordante con el objetivo promulgado en el simposio Ibero-Latinoamericano de Terminología Anatómica, de revisar, discutir y sugerir modificaciones de la terminología vigente para ser presentadas al Comité Federativo sobre Terminología Anatómica (FICAT, 1998) para su consideración de aprobación o desaprobación .

La terminología médica, que incluye la terminología anatómica, se encuentra referida en diccionarios terminológicos. Este lenguaje es internacional y no incluye vocablos comunes, pues con estos es prácticamente imposible referirse con precisión y de forma concisa a un significado, incluso morfológico. Así pues, el texto aceptado mundialmente para la descripción detallada de las distintas estructuras que conforman el cuerpo humano es la Terminologia Anatomica. Sin embargo, en dicho texto no aparece incluido ningún término que permita describir y nominar la pared posterior de los bronquios principales (FICAT), quizá por omisión o por caer erróneamente en una generalización cuando se dice que los bronquios principales tienen una estructura similar a la tráquea. Eventualmente se encuentra algún autor indicando que la pared

\footnotetext{
* Programa de Medicina. Departamento de Ciencias Básicas. Universidad de Caldas, Manizales, Colombia.

** Departamento de Biología. Universidad Autónoma, Manizales, Colombia.

*** Programa de Medicina Veterinaria y Zootecnia. Departamento de Salud Animal. Universidad de Caldas, Manizales, Colombia.
} 
membranosa está prolongada en los bronquios (Latarjet \& Ruíz Liard, 1999). En distintos textos de enseñanza de la anatomía macroscópica se describe que los bronquios principales son la continuación inferior de la tráquea (Testut \& Latarjet, 1949; García-Porrero \& Hurlé, 2005; Dauber, 2007; Standring, 2008; Ellis et al., 2013) y que además poseen elementos de constitución similares a ésta, como los cartílagos bronquiales que presentan forma de herradura (Testut \& Latarjet) y una pared posterior de características fibromembranosas, en la que se incluyen elementos conectivos, musculatura lisa (Testut \& Latarjet; Ferner et al., 2009) y fibras elásticas que se continúan en los bronquios segmentales (Kamel et al., 2009). Además, se ha estudiado mediante técnicas de ultrasonografía la estructura de los bronquios lobares, encontrándose la presencia de tres capas en la región membranosa (Tanaka et al., 2000). No obstante, en ninguno de estos escritos, ni en la Terminologia Anatomica se le da una nominación específica a esa pared fibromembranosa que sella la región posterior de los bronquios.

\section{MATERIAL Y MÉTODO}

Se evaluaron distintos textos clásicos de anatomía macroscópica y artículos de revistas indexadas en los que hicieran alusión a la descripción morfológica de los bronquios principales, con el fin de determinar qué nombre emplean para designar a la membrana fibromuscular que sella la región posterior de los bronquios principales. Luego se contrastó esta información con la contenida para dicha estructura en la Terminologia Anatomica, texto del Federative Committee on Anatomical Terminology que representa todos los aspectos de anatomía y de las asociaciones de anatomistas (FICAT).

\section{RESULTADOS}

Diversos textos de anatomía y artículos de revistas que se evaluaron con relación a la descripción anatómica de los bronquios principales entre ellos (Testut \& Latarjet; Chatain \& Delgado, 1974; Gosling et al., 1990; GarcíaPorrero \& Hurlé; Dauber; Standring; Moore et al., 2010; Pro, 2012; Saladin, 2013) no refieren ni asignan ningún término anatómico que permita describir la pared posterior de tales bronquios. Al confrontar con la Terminologia Anatomica (FICAT) tampoco se encontró ningún término equivalente referido a esa estructura. Se fotografiaron varios especímenes para ratificar la existencia de dicho elemento, el cual aparece sin referencia terminológica.

\section{DISCUSIÓN}

Sólo concebimos pensamientos que podemos expresar con palabras (Banville, 1990), y en la ciencia, como en cualquier otro campo, existe realmente el peligro de intoxicarse con el simbolismo por semejanzas que carecen de significado (Dawkins, 2002), o por dar por sentado hechos que no se han expresado con palabras, lo que lleva a alejarnos cada vez más de la verdad en lugar de acercarnos a ella (Dawkins, 2002). Esto es precisamente lo que sucede con la región posterior de los bronquios, que por caer en una generalización, producto de la comparación de dos estructuras, se omite la descripción de este detalle morfológico.

Dos grandes marcas de contraste del lenguaje científico son la precisión y la claridad (Aparicio \& Saldaña, 2009); la precisión requiere que los términos científicos definan agudamente lo que ellos significan, la claridad es llevada a cabo solamente si en un contexto dado cada término puede ser aplicado exclusivamente a un objeto o fenómeno (Aparicio \& Saldaña), que para nuestro caso corresponde a estos elementos particulares de la vía respiratoria.

El sistema respiratorio de los mamíferos no difiere fundamentalmente (Perry et al., 2000) y los bronquios presentan la misma configuración de la tráquea y su misma estructura y aunque en los reptiles hay presencia de cartílagos anulares, no presentan pared membranosa (Schachner et al., 2013). La caracterización histológica de la pared posterior de los bronquios principales ya ha sido descrita al menos en humanos; sin embargo, la anatomía macroscópica no le ha asignado ningún nombre específico a esta región, como sí lo ha hecho para la tráquea en su porción membranosa con el referente A06.3.01.007 (FICAT).

\section{CONCLUSIÓN}

Siendo consecuentes con el lenguaje científico en cuanto a la precisión y claridad que debe tener cada término empleado, consideramos que el término descriptivo de "pared membranosa" debe ser incluido en la Terminologia Anatomica para designar la región posterior de los bronquios principales. Incluso, dicho término debería hacerse extensivo para aquellos bronquios intrapulmonares que aún presentan estructura semejante a los bronquios principales.

DUQUE, P. J. E.; BARCO, R. J. \& VÉLEZ, G. J. F. Membranous wall bronchus: Proposal for inclusion in the Terminologia Anatomica. Int. J. Morphol., 32(4):1388-1390, 2014. 
SUMMARY: There are two main bronchi breathing tubes originating from the bottom of the trachea and lung projecting to each, allowing passage of air toward and from the bronchioles. The objective of this work was to verify the morphological term inclusion as the back wall of the main bronchus in Anatomic Terminology and texts for teaching gross anatomy. Terminologia Anatomica was analyzed and compared with various texts and journals of gross anatomy teaching, in order to verify if there are descriptions of the posterior wall of the main bronchi and whether it has been nominated. In the Terminologia Anatomica the term for the back wall of the main bronchus is not included. Therefore, it is proposed that the term "membranous wall" for bronchus be included in Terminologia Anatomica.

KEY WORDS: Main Bronchus; Membranous wall; Terminologia Anatomica.

\section{REFERENCIAS BIBLIOGRÁFICAS}

Aparicio, M. A. \& Saldaña, E. Tectotectal neurons and projections: a proposal to establish a consistent nomenclature. Anat. Rec. (Hoboken), 292(2):175-7, 2009.

Banville, J. Copérnico. Madrid, Diario El País, 2005.

Cabré Castellví, M. T.; Estopà-Bagot, R. \& Vargas-Sierra, C. Neology in specialized communication. Terminology, 18(1):1-7, 2012.

Chatain, I. \& Delgado, A. Anatomía Humana. Cali, Universidad del Valle, 1974.

Dauber, W. Pocket Atlas of Human Anatomy. 5th ed. New York, Thieme, 2007.

Dawkins, R. Destejiendo el Arco Iris. Ciencia, ilusión y el deseo de asombro. Barcelona, Tusquets editores, 2002.

Duque, P. J. E.; Gómez Arias, N. C. \& Giraldo Ríos, D. P. Nomenclatura anatómica internacional: ¿un horno microondas en el interior de un volcán activo? Medicina UPB, 21(1):43-55, 2002.

Duque, P. J. E.; Mesa Rincón, V. \& Zapata, G. Reflexiones sobre terminología anatómica, epónimos, medicina y odontología. Arch. Anat. Costa Rica, 5:16-20, 2010.

Ellis, H.; Logan, B. M. \& Dixon, A. K. Cortes anatómicos. Madrid, Marbán, 2013.

Federative International Committee on Anatomical Terminologies (FICAT). Terminologia Anatomica. International Anatomical Terminology. New York, Thieme, 1998.

Ferner, K.; Zeller, U. \& Renfree, M. B. Lung development of monotremes: evidence for the mammalian morphotype. Anat. Rec. (Hoboken), 292(2):190-201, 2009.
García-Porrero, J. A. \& Hurlé, J. M. Anatomía Humana. Madrid, McGraw-Hill Interamericana, 2005.

Gosling, J. A.; Harris, P. F.; Humpherson, J. R.; Whitmore, I. \& Willan, P. L. T. Human Anatomy: Text and Colour Atlas. New York, Gower Medical Publishing, 1990.

Kamel, K. S.; Beckert, L. E. \& Stringer, M. D. Novel insights into the elastic and muscular components of the human trachea. Clin. Anat, 22(6):689-97, 2009.

Latarjet, M. \& Ruíz Liard, A. Anatomía Humana. 3a ed. Madrid, Médica Panamericana, 1999.

Losardo, R. J.; Cruz Gutiérrez, R.; Rodríguez Torres, A.; Prates, J. C. \& Prates, N. E. V. R. Iberia-Latin-American Symposia of Morphological Terminology (SILAT). First Two Years and Statute. Int. J. Morphol, 28(4):1323-6, 2010.

Moore, K. L.; Dalley, A. F. \& Agur, A. M. R. Anatomía con orientación clínica. Barcelona, Wolters Cluwer Lippincot Williams \& Wilkins, 2010.

Perry, S. F.; Schmitz, A.; Andersen, N. A.; Wallau, B. R. \& Nicol, S. Descriptive study of the diaphragm and lungs in the short-nosed echidna, Tachyglossus aculeatus (Mammalia: monotremata). J. Morphol., 243(3):247-55, 2000.

Pró, E. A. Anatomía clínica. Buenos Aires, Médica Panamericana, 2012.

Saladin, K. S. Anatomía y Fisiología: la unidad entre forma y función. 6a ed. México D.F., McGraw-Hill Education, 2013.

Schachner, E. R.; Hutchinson, J. R. \& Farmer, C. G. Pulmonary anatomy in the Nile crocodile and the evolution of unidirectional airflow in Archosauria. PeerJ.,1:e60, 2013.

Standring, S. Gray's Anatomy. The Anatomical basis of clinical practice. Philadelphia, Churchill Livingstone, 2008.

Tanaka, F.; Muro, K.; Yamasaki, S.; Watanabe, G.; Shimada, Y.; Imamura, M.; Hitomi, S. \& Wada, H. Evaluation of tracheobronchial wall invasion using transbronchial ultrasonography (TBUS). Eur. J. Cardiothorac. Surg., 17(5):570-4, 2000.

Testut, L. \& Latarjet, A. Tratado de anatomía humana. Barcelona, Salvat, 1949.

\section{Dirección para Correspondencia: \\ Jorge Eduardo Duque Parra \\ Programa de Medicina \\ Universidad de Caldas, Manizales \\ COLOMBIA}

Email: jorge.duque_p@ucaldas.edu.co

Recibido : 19-06-2014

Aceptado: 28-08-2014 